\title{
AVALIAÇÃO FENOTÍPICA, GENOTÍPICA E DESEMPENHO DE ESTABILIDADE EM CULTIVARES DE BANANEIRAS NO AMAPÁ
}

\author{
Gilberto Ken Iti Yokomizo ${ }^{1}$, Jurema do Socorro Azevedo Dias ${ }^{1}$, João Batista Duarte ${ }^{2}$ \\ ${ }^{1}$ Embrapa Amapá, E-mail: gilberto.yokomizo@embrapa.br, jurema.dias@embrapa.br \\ ${ }^{2}$ Universidade Federal de Goiás, E-mail: jbduarte@ufg.br
}

\section{RESUMO}

Os objetivos deste trabalho foram verificar a estabilidade, as correlações e os parâmetros fenotípicos e genotípicos nas cultivares Fhia-1, Fhia-18, PV-0344, Thap Maeo e Caipira em características vegetativas, produtivas e de desenvolvimento da Sigatoka-negra. Após três ciclos os resultados indicaram presença de maior contribuição genética nas características avaliadas, exceto em severidade da doença na décima folha (SEV10), folha mais jovem infectada e número de folhas viáveis (NFV), com maior contribuição ambiental e da interação genótipos com anos; plantas mais vigorosas e desenvolvidas demonstraram ser mais produtivas, pois apresentaram correlações entre as características vegetativas e de produção positivas, enquanto as com SEV10 foram negativas. Pode-se concluir que as características de doença e NFV dificultam a seleção; a severidade da doença prejudica todas as demais características; as cultivares mais estáveis foram a Thap Maeo e a Caipira, enquanto Fhia-1 e PV-0344 foram mais suscetíveis para o desenvolvimento da doença.

Palavras-chave: Musa spp., Estabilidade de Wricke, Seleção, Ecovalência

\section{PHENOTYPIC, GENOTYPIC EVALUATION AND PERFORMANCE OF STABILITY OF THE BANANA CULTIVARS IN AMAPA STATE, BRAZIL}

\begin{abstract}
The objectives of this study were to verify the stability, the correlations and the phenotypic and genotypic parameters in the cultivars FHIA-1 FHIA-18, PV-0344, Thap Maeo and Caipira. After three cycles the results indicated the presence of higher genetic contribution to the characteristics evaluated, except in disease severity in the number ten leaf (SEV10), number of the youngest leaf infected and number of viable leaves (NVL) with higher environmental contribution and genotype and years interaction; more vigorous and developed plants demonstrated to be more productive, with positive correlations between vegetative and production characteristics, while with SEV10 they were negative. One concluded that the characteristics related to the disease and NVL made difficult the selection; the disease severity damages all the other characteristics; the
\end{abstract}


most stable cultivars were Thap Maeo and Caipira while FHIA-1 and PV-0344 were more susceptible to the disease development.

Keywords: Musa spp., Wricke stability, Selection, Ecovalence

\section{INTRODUÇÃO}

A bananeira (Musa spp.) é cultivada em mais de 150 países tropicais e subtropicais, com produção de 105 a 120 milhões de toneladas de frutas frescas, numa área colhida de quatro milhões de hectares (UNCTAD, 2015). O Brasil é o segundo maior produtor mundial, produzindo entre 7,18 e 7,33 milhões de toneladas, gerando US\$ 997 milhões em uma área cultivada de 504.248 hectares (IBGE, 2014; FAOSTAT, 2015). O mercado interno brasileiro consome quase a totalidade de sua produção (FAOSTAT, 2015), tornando-se interessante aumentar sua produtividade para exportação.

Aspectos relacionados à fitossanidade têm sido um dos principais entraves na bananicultura (SILVA et al., 2002), devido à baixa diversidade genética refletindo em poucas cultivares disponíveis, abrangendo principalmente as cultivares Prata e Maçã, suscetíveis a diversas doenças, como a Sigatoka-Negra, causada por Mycosphaerella fijiensis Morelet. Desta forma, nos programas de melhoramento genético desta frutífera, além de resistência às principais pragas e doenças, deve-se tentar associar num mesmo genótipo, porte adequado, boa produtividade, estabilidade e adaptação aos diferentes ecossistemas, além de atender ao paladar dos consumidores (DONATO et al., 2006; RODRIGUES et al., 2006).

Adicionalmente, existe também a interação de genótipos com os efeitos ambientais (GxA), que torna necessária a obtenção de informações pormenorizadas sobre o comportamento desses genótipos nas localidades onde se deseja cultivá-los (CRUZ et al., 2012). Os fatores como água, salinidade, vento ou temperatura podem gerar estresses abióticos e, com isso, fazer com que a planta apresente alta instabilidade em diversas características. A bananeira tem apresentado muitos desafios frente a estresses bióticos e abióticos desse tipo, necessitando urgentemente de pesquisas que possam melhorar a eficiência e estabilidade de sua produção (HESLOP-HARRISON \& SCHWARZACHER, 2007).

Existem vários métodos de análise da estabilidade fenotípica para esse tipo de estudo (AMORIM et al., 2006). Estes diferenciam-se quanto aos conceitos de seus parâmetros de estabilidade, o número deles, 
os procedimentos biométricos para sua estimação e nas exigências para utilização de cada um. Para estudos de estabilidade, Borém \& Miranda (2009) destacam a estatística "ecovalência", de Wricke (1965), como de fácil aplicação e entendimento. A estimação desse parâmetro é baseada na decomposição da soma de quadrados da interação GxA, em partes associadas à contribuição de cada genótipo para essa interação. Assim, identificam-se aqueles de maior estabilidade com menor contribuição para GxA, os quais poderiam ser recomendados amplamente para a região alvo do estudo.

Trabalhos visando a estimar parâmetros de estabilidade com a cultura da bananeira são poucos (CAUWER \& ORITZ, 1998; CAUWER et al., 1995; ORTIZ \& CAUWER, 1998). Ademais, os estudos disponíveis têm sido realizados em condições diversas às observadas em regiões produtoras no Brasil. Logo, justificam-se pesquisas desse tipo nas diferentes regiões produtoras de banana do país.

Os objetivos deste trabalho foram estimar informações referentes à estabilidade de cultivares de bananeiras, em três anos de cultivo, verificar a presença de variabilidade genética entre elas, bem como estimar parâmetros fenotípicos e genotípicos, e correlações entre características vegetativas, produtivas e de manifestação de SigatokaNegra.

\section{MATERIAL E MÉTODOS}

Um experimento foi conduzido em área de produção e avaliado entre 2003 a 2005, na Colônia Agrícola do Matapi, no município de Porto Grande, AP, Brasil. O clima predominante é do tipo Ami, caracterizado como tropical chuvoso. A temperatura média anual é de $27^{\circ} \mathrm{C}$, com variação de temperatura sempre inferior a $5^{\circ} \mathrm{C}$. O regime pluviométrico anual define uma estação relativamente seca, porém, com pluviosidade total acima de $1.900 \mathrm{~mm}$. O estudo foi desenvolvido em ecossistema de Terra Firme, em meio à vegetação do tipo Floresta equatorial subperenifólia. (OLIVEIRA JÚNIOR \& MELÉM JÚNIOR, 2000).

Os tratamentos foram constituídos pelas cultivares Caipira, Thap Maeo, PV0344, FHIA-01 e FHIA-18. O espaçamento utilizado entre plantas foi de $3 \mathrm{~m} \times 3 \mathrm{~m}$, com delineamento experimental em blocos casualizados e quatro repetições. Cada repetição foi constituída por nove plantas, descartando-se as três de cada extremidade e considerando-se como úteis as três plantas situadas no centro da parcela. 


\section{AVALIAÇÃO FENOTÍPICA, GENOTÍPICA E DESEMPENHO DE ESTABILIDADE EM CULTIVARES \\ DE BANANEIRAS NO AMAPÁ}

As avaliações foram realizadas para as seguintes características: severidade da doença na décima folha (SEV10); número da folha mais jovem infectada (FMJI); altura da planta do nível do solo à inserção da inflorescência (APL), em metros; circunferência do pseudocaule a $30 \mathrm{~cm}$ de altura do solo (CPL), em centímetros; número de folhas viáveis (NFV); peso médio de cachos da parcela (PCA), em kg; peso médio das pencas dos cachos da parcela (PPE), em kg; e número médio de pencas presentes nos cachos da parcela (NPE).

Para a obtenção dos valores da severidade da doença (SEV10) utilizou-se uma escala diagramática com sete graus, em função da percentagem de área foliar lesionada, segundo Stover \& Dickson (1970), modificada por Gauhl et al. (1993) e Gasparotto et al. (2006) citados por Dias \& Barreto (2011). Após a coleta de dados, calculou-se a Área Foliar total atacada por planta pelo fungo, utilizando-se da seguinte fórmula:

$\operatorname{AFA}(\%)=\frac{\sum(\mathrm{LN} 1 \times 1+\ldots \mathrm{LN} 6 \times 100)}{\mathrm{LN}}$

em que: AFA $(\%)=$ Área Foliar Total Atacada; LN1 a LN6 = no de folhas com o respectivo grau em percentagem; $L N=n^{\circ}$ de folhas.
A ecovalência de Wricke $\left(\omega_{i}\right)$, foi estimada por meio da equação $\omega_{i}=\sum_{j=1}^{n}(\text { ge })_{i j}^{2}$, com $(\text { ge })_{i j}=Y_{i j}-\bar{Y}_{i .}-\bar{Y}_{. j}+\bar{Y}_{. .}$, em que $Y_{i j}$ é a média da cultivar “i” no ano “j”; $\bar{Y}_{i .}$ é a média da cultivar "i" em todos os anos; $\bar{Y}_{. j}$ é a média do ano "j" para todos as cultivares e $\bar{Y}$.é a média geral. O somatório dos valores $\omega_{\mathrm{i}}$ corresponde ao valor da soma dos quadrados da interação GxA. Dessa forma, é possível calcular a porcentagem da interação GxA devido a cada cultivar $\left(\omega_{\mathrm{i}} \%\right)$, pela equação: $\omega_{\mathrm{i}} \%=\left(\underset{\mathrm{i}}{\omega_{\mathrm{i}} / \sum_{\mathrm{i}} \omega_{\mathrm{i}}}\right) 100 \cdot$ Quanto menores os valores de $\omega_{i}$ e $\omega_{i} \%$, mais estáveis são as correspondentes cultivares.

Para a análise de variância conjunta entre anos, estimou-se a relação entre o maior e o menor QM do resíduo das análises de variância individuais, adotando-se o critério de que relações próximas ou abaixo de sete (PIMENTEL GOMES, 2009) são indicadoras de homogeneidade de variâncias (homocedasticia). Nestas condições, a análise conjunta pode ser realizada sem necessidade de ajuste nos graus de liberdade das fontes de variação.

A escala adotada para as correlações de Pearson foi semelhante à citada por Santos (2007), considerando correlações entre $0 \%$ a 
$10 \%$ como baixas; $10 \%$ a $50 \%$ como médias baixas; $50 \%$ a $80 \%$ como médias altas e acima de $80 \%$ como altas, em concordância com Cohen (2013) que cita que os limites são geralmente subjetivos e devem ser flexíveis, conforme o contexto e o propósito da pesquisa.

Todas estas análises foram realizadas com auxílio do programa Genes (CRUZ, 2006).

\section{RESULTADOS E DISCUSSÃO}

Antes de se realizar a análise de variância conjunta é necessário verificar se existe homogeneidade entre os quadrados médios dos resíduos de cada ano. A relação entre o maior e o menor QM do resíduo, para as diferenes características (Tabela 1), com valores próximos ou abaixo de sete, demonstra que as variâncias residuais relativas aos experimentos envolvidos na análise foram homogêneas e a precisão experimental entre os ensaios (três anos) não foi discrepante. Desta forma, pode-se realizar a análise conjunta sem restrições conforme preconiza Pimentel Gomes (2009), sem a necessidade de ajuste nos graus de liberdade para as características.

No resumo da análise de variância conjunta (Tabela 2) pode-se observar que as cultivares de bananeira foram diferentes estatisticamente, tanto em características vegetativas (altura e circunferência do pseudocaule da bananeira), como na produção (peso e número de frutos). Silva et al. (2002), Ledo et al. (2008), Lessa et al. (2010) e Borges et al. (2011) também obtiveram a presença de variabilidade nestas mesmas características em experimentos com bananeiras.

Os anos também foram responsáveis por diferenças significativas em todas as características, exceto em peso de cacho e de pencas. $\mathrm{Na}$ fonte de variação da interação Cultivares $x$ Anos pode-se observar que apenas nas características número de folhas viáveis (NFV) e altura da planta (APL) não houve diferenças, diferindo do observado por Roque et al. (2014), indicando menor intensidade de seus efeitos sobre a manifestação fenotípica final, ou seja, estas duas características foram menos influenciadas pelos anos. Para a característica PPE, enquanto aqui houve alta interação, ou seja, elevada influência de anos, Roque et al. (2014) obtiveram pouca influência.

Os CVs experimentais foram baixos para as características vegetativas, entre $6,63 \%$ a $11,55 \%$, e médios para aquelas relacionadas à produção, variando de 13,58\% a $23,33 \%$. Os maiores valores foram 
observados nas características peso e número de frutos. Apesar disso, por se referirem a aspectos de produção estas características podem ser consideradas aceitáveis, sendo que todos os valores, excetuando-se NFV, foram superiores aos observados por Donato et al. (2006) e Lessa et al. (2010); possivelmente devido à maior amplitude de variações ambientais na região alvo do presente estudo.

Tabela 1. Quadrados médios dos resíduos (QMr) das análises individuais e relação ${ }^{\mathrm{b}}$ entre $\mathrm{QMr}_{\text {maior }} / \mathrm{QMr}$ menor para as características: SEV10: severidade da doença na décima folha; FMJI: número da folha mais jovem infectada; APL: altura da planta; CPL: circunferência da planta; NFV: número de folhas vivas; PCA: peso do cacho; PPE: peso médio das pencas; NPE: número de pencas presentes no cacho; avaliadas em cultivares de bananeira em três anos (Macapá, AP), 2003 a 2005.

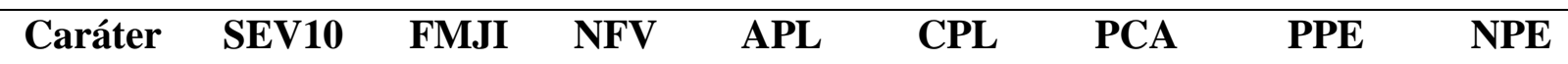

\begin{tabular}{ccccccccc}
\hline Ano & & & & & & & & \\
$\mathbf{1}$ & 191,06 & 3,12 & 1,09 & 0,02 & 11,46 & 5,77 & 4,70 & 0,39 \\
$\mathbf{2}$ & 135,41 & 8,37 & 1,12 & 0,09 & 26,30 & 11,96 & 12,95 & 2,82 \\
$\mathbf{3}$ & 38,46 & 4,49 & 3,45 & 0,08 & 12,35 & 8,54 & 7,63 & 0,38 \\
\hline Relação & 4,97 & 2,69 & 3,17 & 4,25 & 2,29 & 2,07 & 2,75 & 7,51 \\
\hline
\end{tabular}

Tabela 2. Resumo da análise de variância para as características: SEV10: severidade da doença na décima folha; FMJI: número da folha mais jovem infectada; APL: altura da planta; CPL: circunferência da planta; NFV: número de folhas vivas; PCA: peso do cacho; PPE: peso médio das pencas; NPE: número de pencas presentes no cacho; avaliadas em cultivares de bananeiras em três anos (Macapá, AP), 2003 a 2005.

\begin{tabular}{lccccccccc}
\hline \multicolumn{1}{c}{ FV } & GL & SEV10 & FMJI & \multicolumn{1}{c}{ NFV } & APL & CPL & PCA & PPE & NPE \\
\hline Trat (T) & 4 & $627,88^{* *}$ & $9,46^{\text {ns }}$ & $3,87^{\text {ns }}$ & $1,065^{* *}$ & $763,48^{* *}$ & $258,49^{* *}$ & $239,16^{* *}$ & $32,20 * *$ \\
Anos (A) & 2 & $4463,86^{* *}$ & $105,36^{* *}$ & $53,08^{* *}$ & $6,282^{* *}$ & $1611,74^{* *}$ & $29,22^{\text {ns }}$ & $32,75^{\text {ns }}$ & $13,96 * *$ \\
TxA & 8 & $395,04^{* *}$ & $15,44^{*}$ & $2,49^{\text {ns }}$ & $0,106^{\text {ns }}$ & $50,26^{*}$ & $33,33^{* *}$ & $30,81^{*}$ & $8,68 * *$ \\
Resíduo & 45 & 108,23 & 6,58 & 1,85 & 0,063 & 20,48 & 11,13 & 10,28 & 1,34 \\
\hline Total & 59 & & & & & & & & \\
\hline Média & & 15,70 & 4,05 & 11,79 & 2,86 & 68,24 & 15,52 & 14,32 & 8,52 \\
CV\% & 66,26 & 63,35 & 11,55 & 8,79 & 6,63 & 21,50 & 22,39 & 13,58 \\
\hline
\end{tabular}


As estimativas dos parâmetros genéticos e fenotípicos (Tabela 3) indicam que o comportamento das cultivares teve maior contribuição genética, excetuando-se para as características SEV10, FMJI e NFV, nas quais houve maiores contribuições da interação GxA e da variação residual, indicando que estas são mais influenciadas pelas condições ambientais.

As características que apresentaram valores altos de coeficientes de determinação são as que têm maior contribuição genética para o fenótipo e, desta forma, maior possibilidade de manifestação em gerações posteriores. Mas também houve aquelas características com alta influência ambiental, como SEV10, FMJI e NFV. Os coeficientes de determinação genotípica, segundo Resende (2002), podem ser classificados como baixos $\left(10<\mathrm{R}_{\mathrm{G}}^{2} \%<40\right)$, médios ou moderados $\left(40 \leq \mathrm{R}^{2}{ }_{\mathrm{G}} \% \leq 70\right)$ e altos $\left(\mathrm{R}^{2}{ }_{\mathrm{G}} \%>\right.$ 70) e, utilizando-se destas classes, todos as características apresentaram valores altos, excetuando-se os caracteres FMJI e NFV, cujas estimativas foram classificadas como baixa e média, respectivamente. Isso significa que há variações nos fenótipos apresentados, conforme diferentes fatores ambientais lhes são impostos.

Tabela 3. Parâmetros genéticos e fenotípicos para as características: SEV10: severidade da doença na décima folha; FMJI: número da folha mais jovem infectada; APL: altura da planta; CPL: circunferência da planta; NFV: número de folhas vivas; PCA: peso do cacho; PPE: peso médio das pencas; NPE: número de pencas presentes no cacho; avaliadas em cultivares de bananeiras em três anos (Macapá, AP), 2003 a 2005.

\begin{tabular}{lrrrrrrrr}
\hline Parâmetros $^{\mathbf{b}}$ & SEV10 & FMJI & NFV & APL & CPL & PCA & PPE & NPE \\
\hline $\boldsymbol{\sigma}^{2} \mathbf{g}$ & 43,30 & 0,240 & 0,168 & 0,083 & 61,92 & 20,61 & 19,07 & 2,57 \\
$\boldsymbol{\sigma}^{2} \mathbf{g x a}$ & 71,70 & 2,214 & 0,160 & 0,011 & 7,44 & 5,55 & 5,13 & 1,83 \\
$\boldsymbol{\sigma}^{2} \mathbf{r}$ & 108,23 & 6,583 & 1,853 & 0,063 & 20,48 & 11,13 & 10,28 & 1,34 \\
$\mathbf{R}^{2} \mathbf{g} \%$ & 82,76 & 30,41 & 52,13 & 94,09 & 97,32 & 95,69 & 95,70 & 95,84 \\
$\mathbf{C V g}(\boldsymbol{\%})$ & 41,92 & 12,09 & 3,48 & 10,12 & 11,53 & 29,25 & 30,51 & 19,82 \\
$\mathbf{C V g} / \mathbf{C V e}$ & 0,63 & 0,19 & 0,30 & 1,15 & 1,74 & 1,36 & 1,36 & 1,39
\end{tabular}

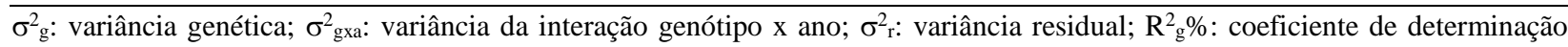
genético em porcentagem; CVg (\%): coeficiente de variação genético, em porcentagem; CVg/CVe: relação do coeficiente de variação genético/coeficiente de variação ambiental. 
Para as características APL, CPL, PCA, PPE e NPE os valores da variação genética são indicativos de variabilidade suficiente para efetiva seleção de materiais superiores.

A relação $\mathrm{CVg} / \mathrm{CVe}$ é um indicador da facilidade em se realizar a seleção e obter êxito com menores esforços e uso de procedimentos estatísticos menos complexos. Valores próximos ou acima da unidade demonstram grande chance de se obter sucesso.

Apesar de ter sido obtido um coeficiente de variação genética aparentemente alto para SEV10, não houve correspondência favorável na estimativa de CVg/CVe. Já para SEV, FMJI e NFV foram obtidas estimativas abaixo da unidade, indicativo de que serão necessárias análises mais apuradas para inferir sobre o seu potencial de uso no processo de seleção. Relações interessantes foram observadas para produtividade em peso e número de frutos (estimativas entre 1,15 e 1,74), ou seja, há variabilidade e relativa facilidade de se selecionar genótipos superiores.

As correlações entre as características avaliadas foram todas positivas, excetuandose aquelas envolvendo SEV10, sendo que apenas a correlação entre SEV10 com FMJI foi significativa e as demais envolvendo a característica SEV10 estiveram entre média baixa e alta (Tabela 4$)$.

Tabela 4. Coeficientes de correlações de Pearson em porcentagem para as características: SEV10: severidade da doença na folha 10; FMJI: número da folha mais jovem infectada; APL: altura da planta; CPL: circunferência da planta; NFV: número de folhas vivas; PCA: peso do cacho; PPE: peso médio das pencas; NPE: número de pencas presentes no cacho; avaliadas em cinco cultivares de bananeiras (Macapá, AP), 2003 a 2005.

\begin{tabular}{cccccccc}
\hline & SEV10 & FMJI & NFV & APL & CPL & PCA & PPE \\
\hline FMJI & $-87,97 *$ & & & & & \\
NFV & $-57,54$ & 39,65 & & & & & \\
APL & $-39,65$ & 49,30 & 16,86 & & & & \\
CPL & $-72,71$ & 50,20 & $96,57 * *$ & 30,55 & & & \\
PCA & $-62,45$ & 35,48 & $97,13 * *$ & 5,74 & $96,68 * *$ & & \\
PPE & $-62,77$ & 37,62 & $98,17 * *$ & 9,22 & $97,43 * *$ & $99,87 * *$ & \\
NPE & $-46,15$ & 29,95 & 82,30 & 61,48 & 85,85 & 75,61 & 77,72 \\
\hline$* * *:$ Significativo a 1\% e 5\% de probabilidade, pelo teste t. & & & &
\end{tabular}

**,* : Significativo a $1 \%$ e $5 \%$ de probabilidade, pelo teste t. 
Valores negativos dos coeficientes de correlação de Pearson entre as características vegetativas e de produção com aquelas que quantificam danos às plantas, neste caso a severidade da doença são esperados, pois com o aumento dos sintomas da doença, destruindo área fotossintética das folhas, há prejuízos fisiológicos na planta. Isso é confirmado com os resultados obtidos (Tabela 4), pois tanto FMJI e SEV10 apresentaram correlações negativas com as demais características avaliadas.

As correlações entre características vegetativas e de produção foram positivas, destacando-se as que envolveram o número de folhas viáveis (NFV) com CPL, PCA e PPE; pois com acréscimos nesta característica há maiores desenvolvimento do caule, peso dos cachos e peso de pencas, por haver aumento na capacidade fotossintética da planta. $O$ peso de cacho teve correlação positiva e alta com a circunferência do caule. Isso significa que plantas com melhor estrutura tendem a apresentar cachos maiores e também induzem diretamente a formação de pencas em número maior e mais pesadas. Comportamento semelhante ao citado por Ledo et al. (2008), de que genótipos com maior número de frutos/cacho e com maior peso médio apresentarão maior produção por cacho, correspondendo a correlações positivas entre PCA, PPE e NPE, e contrário ao de Lima et al. (2005) cujos resultados de correlações foram diferentes, sendo que, especificamente, o grupo de características NFV, CPL, PCA e PPE foram as que apresentaram correlações positivas e altas entre si.

O método da ecovalência, devido à pesquisa ter sido realizada com a avaliação de três épocas, mostrou-se adequado para esse tipo de estudo, pois é indicado nos casos em que a avaliação engloba poucos genótipos e ambientes/anos (CRUZ et al., 2012). E, também, é conveniente para avaliar a estabilidade temporal em determinada localidade (ROCHA et al., 2003), o que foi um dos objetivos deste trabalho. Inexistem estudos que tenham utilizado a ecovalência em bananeiras, justificando a importância da divulgação dos resultados aqui obtidos, principalmente quanto à visualização do comportamento de cultivares de bananeira em condições tropicais, como as existentes no Amapá. Além disso, essa estatística é um parâmetro de fácil interpretação para comparação do desempenho de cultivares, quanto à estabilidade fenotípica. As cultivares que apresentaram maiores valores de ecovalência para cada característica são consideradas como as menos estáveis (Tabela 5). 
Tabela 5. Avaliação da interação GxA para as características: SEV10: severidade da doença na décima folha; FMJI: número da folha mais jovem infectada; APL: altura da planta; CPL: circunferência da planta; NFV: número de folhas vivas; PCA: peso do cacho; PPE: peso médio das pencas; NPE: número de pencas presentes no cacho; pela ecovalência em porcentagem (Wi\%) estimada pelo teste de Wricke para as médias de cinco cultivares de bananeiras em três anos em conjunto (Macapá, AP), 2003 a 2005.

\begin{tabular}{|c|c|c|c|c|c|c|c|c|c|c|c|c|}
\hline Caracteres & SEV10 & FMJI & NFV & APL & CPL & PCA & PPE & NPE & PCA & PPE & $\mathbf{P C A}^{\mathbf{a}, \mathrm{b}}$ & $\mathbf{P P E}^{\mathbf{a}, \mathrm{b}}$ \\
\hline Fhia-1 & 45,22 & 23,46 & 0,71 & 12,80 & 0,09 & 3,02 & 1,16 & 68,01 & 22,41 & 20,89 & $22,41 \mathrm{a}$ & $20,89 a$ \\
\hline Fhia-18 & 6,20 & 61,85 & 5,94 & 54,70 & 6,65 & 55,26 & 58,34 & 4,45 & 16,96 & 15,61 & $16,96 a b$ & $15,61 \mathrm{ab}$ \\
\hline PV-0344 & 43,43 & 13,18 & 46,23 & 9,02 & 49,04 & 38,92 & 35,69 & 5,81 & 11,52 & 10,16 & $11,52 b$ & $10,16 b$ \\
\hline $\begin{array}{l}\text { Thap } \\
\text { Maeo }\end{array}$ & 1,32 & 0,53 & 17,37 & 2,41 & 10,35 & 1,88 & 1,38 & 8,05 & 15,73 & 14,78 & $15,73 \mathrm{ab}$ & $14,78 \mathrm{ab}$ \\
\hline Caipira & 3,83 & 0,98 & 29,74 & 21,07 & 33,87 & 0,91 & 3,43 & 13,68 & 10,99 & 10,14 & $10,99 b$ & $10,14 b$ \\
\hline
\end{tabular}

a Médias das produtividades em kg para as características peso do cacho (PCA) e peso médio das pencas (PPE)

b Médias seguidas pela mesma letra, na mesma coluna, não diferem entre si, ao nível de 5\% de probabilidade, pelo teste de Tukey.

Desta forma, a cultivar Fhia-1 apresentou alta contribuição para a interação GxA nas características SEV10 e NPE; a cultivar Fhia-18 para FMJI, APL, PCA e PPE; a cultivar PV-0344 para SEV10, NFV, CPL, PCA e PPE; e a cultivar Caipira para NFV e CPL.

As cultivares Thap Maeo e Caipira foram as mais estáveis, enquanto Fhia-8 e PV-0344, com maiores contribuições de ecovalência, foram as menos estáveis. Já Fhia 1, entre as cultivares avaliadas, mostrou estabilidade intermediária (Tabela 5). Com este comportamento pode-se afirmar que as cultivares Thap Maeo e Caipira, mais estáveis, são menos responsivas a fatores ambientais específicos; ressaltando-se, porém, que a primeira teve o melhor desempenho em produção.

As cultivares Fhia-18 e PV-0344, considerando-se suas maiores contribuições para a interação GxA, foram as menos estáveis nas características avaliadas, mostrando-se mais responsivas a fatores específicos de ambiente, principalmente em relação às melhorias na insolação, fornecimento de água e nutrientes. Torna-se conveniente dar atenção a estas cultivares, com a primeira apresentando melhor desempenho de produção, já que mostraram contribuições substanciais para SEV10 e FMJI, que são características relacionadas à 
manifestação dos sintomas da SigatokaNegra, sendo responsáveis por quase $50 \%$ da manifestação da doença (SEV10), o que é indesejável. Entre as cultivares avaliadas, Fhia-1 teve desempenho intermediário de estabilidade, considerando-se todas as características, embora destacando-se com a maior média de produção. Cabe ressalva, porém, quanto à sua alta contribuição em SEV10. Isso indica que em algumas situações o material mais estável pode não ser o mais produtivo, e aquele com maior suscetibilidade pode fisiologicamente contrabalançar os danos causados pela doença com resposta em produção. Similarmente ao que se observou no presente estudo, Lessa et al. (2014) citam que há tendência de estabilização da produção a partir do segundo ciclo de avaliações.

\section{CONCLUSÕES}

As características vegetativas e de produção foram as que apresentaram efeitos genotípicos superiores, revelando maior facilidade para seleção. Ocorre o oposto para as características relacionadas à doença, que demonstraram possuir maior contribuição dos efeitos de anos e da interação genótipo $\mathrm{x}$ ambiente.

As características vegetativas e de produção respondem de forma negativa ao aumento da severidade da doença, com base nas correlações encontradas, indicando que as cultivares avaliadas possuem pouca tolerância ou ausência de resistência.

Quanto maior foi a capacidade fotossintética (desenvolvimento vegetativo) houve maior resposta de produção, com isso plantas mais vigorosas e desenvolvidas demonstram ser mais produtivas, ou seja, não há presença de compensação fisiológica.

As cultivares mais estáveis foram a Thap Maeo e a Caipira, sendo que as com maior contribuição para o desenvolvimento da doença foram as cultivares Fhia-1 e PV0344, com base na análise de ecovalência.

\section{REFERÊNCIAS}

AMORIM, E.P.; CAMARGO, C.E.O.; FERREIRA FILHO, A.W.P.; PETTINELLI JUNIOR, A.; GALLO, P.B.; AZEVEDO FILHO, J.A. 2006. Adaptabilidade e estabilidade de linhagens de trigo no Estado de São Paulo. Bragantia, Campinas, v.65, p.575-582.

BORÉM, A.; MIRANDA, G. V. 2009. Melhoramento de Plantas. 5. ed. Viçosa: Editora UFV, v.1, 529p.

BORGES, R. S.; SILVA, S. O.; OLIVEIRA, F.T.; ROBERTO, S.R. 2011. Avaliação de genótipos de bananeira no norte do Estado do Paraná. Revista Brasileira de Fruticultura, Jaboticabal, v.33, p. 291296.

CAUWER, I.; ORTIZ, R. 1988. Analysis of the genotype $\mathrm{x}$ environment interaction in musa trials. Experimental Agriculture, Cambridge, v.34, p.177-188. 
CAUWER, I.; VUYLSTEKE, D.; ORTIZ, R. 1995. Yield Nigeria and Cameroon. MusAfrica, IITA, Nigéria, v.6, p.15-16.

COHEN, J. 2013. Statistical power analysis for the behavioral sciences. Londres: Routledge, 2. ed revisada, $567 \mathrm{p}$.

CRUZ, C.D.; REGAZZI, A.J.; CARNEIRO, P.C.S. 2012. Modelos biométricos aplicados ao melhoramento genético. Viçosa: Editora UFV, 514 p.

CRUZ, C.D. 2006. Programa Genes Biometria. Viçosa: Editora UFV, 382p.

DONATO, S.L.R.; SILVA, S.O.; LUCCA FILHO, O.A.; LIMA, M.B.; DOMINGUES, H.; ALVES, J.S. 2006. Correlação entre caracteres da planta e do cacho em bananeira (Musa spp.). Ciência e Agrotecnologia, Lavras, v.30, p.21-30.

FAOSTAT 2011. Food Balance Sheets. Disponível em: <http://faostat3.fao.org/download/FB/FB S/E >. Acesso em: 05 jan. de 2015.

HESLOP-HARRISON, J. S.; SCHWARZACHER, T. 2007. Domestication, Genomics and the Future for Banana. Annals of Botany, Oxford, v.100, p.1073-1084.

HOOGERHEIDE, E.S.S.; FARIAS, F.J.C.; VENCOVSKY, R.; FREIRE, E.C. 2007. Estabilidade fenotípica de genótipos de algodoeiro no estado do Mato Grosso. Pesquisa Agropecuária Brasileira, Brasília, v.42, p.695-698.

IBGE. Levantamento Sistemático da Produção Agrícola. 2014. IBGE, Rio de Janeiro, v.27, 86p.

LEDO, A. S.; SILVA JUNIOR, J.F.; LÉDO, C.A. S.; SILVA, S. O. 2008. Avaliação de genótipos de bananeira na região do Baixo São Francisco, Sergipe. Revista Brasileira de Fruticultura, Jaboticabal, v.30, p.691-695.

LESSA, L. S.; LEDO, C. A. S.; SANTOS, V. S.; SILVA, S. O.; PEIXOTO, C.P. 2010. Seleção de híbridos diplóides (AA) de bananeira com base em três índices não paramétricos. Bragantia, Campinas, v.69, p.525-534.

LESSA, L. S.; LEDO, C. A. S.; AMORIM, E. P.; SILVA, S. O. 2014. Estimativa de repetibilidade de híbridos diploides (AA) de bananeira. Pesquisa Agropecuária Brasileira, Brasília, v.49, p.109-117.

LIMA, M.B.; SILVA, S. O.; JESUS, O.N.; OLIVEIRA, W.S.J.; AZEVEDO, R.L. 2005. Avaliação de cultivares e híbridos de bananeira no Recôncavo Baiano. Ciência e Agrotecnologia, Lavras, v.29, p.515-520.

OLIVEIRA JÚNIOR, R.C.; MELÉM JÚNIOR, N.J. 2000. Zoneamento agroecológico do município de Porto Grande: relatório final. Embrapa Amapá, Macapá, 68p.

ORTIZ, R.; CAUWER, I. 1998. Genotypeby-environment interaction and testing environments for Plantain and Banana (Musa spp. L.) breeding in West Africa. Tropicultura, Bruxelas, v.16, p.97-102.

PIMENTEL GOMES, F. 2009. Curso de estatística experimental. 15. ed. Fealq, Piracicaba, 451p.

RESENDE, M.D.V. 2009. Genética Biométrica e estatística no melhoramento de plantas perenes. Colombo: Embrapa Florestas, 975p.

ROCHA, M.M.; VELLO, N.A.; LOPES, A.C.A.; MAIA, C.C.M. 2003. Comportamento produtivo de genótipos de soja no município de Piracicaba, São Paulo. Boletim de Pesquisa 48, Embrapa Meio Norte, Teresina, Piauí, $19 \mathrm{p}$.

RODRIGUES, M.G.V.; SOUTO, R.F.; SILVA, S. O. 2006. Avaliação de genótipos de bananeira sob irrigação. Revista Brasileira de Fruticultura, Jaboticabal, v.28, p.444-448.

ROQUE, R. L.; AMORIM, T.B.; FERREIRA, C.F.; LEDO, C.A.S.; AMORIM, E.P. 2014. Desempenho 
agronômico de genótipos de bananeira no Recôncavo da Bahia. Revista Brasileira de Fruticultura, Jaboticabal, v.36, n.3, p.598- 609.

SANTOS, C. 2007. Estatística Descritiva Manual de Auto-aprendizagem. Lisboa: Edições Silabo, 264 p.

SILVA, S.O; FLORES, J.C.O.; LIMA NETO, F.P. 2002. Avaliação de cultivares e híbridos de bananeira em quatro ciclos de produção. Pesquisa Agropecuária Brasileira, Brasília, v.37, p. $1567-1574$.

UNCTAD, 2015 Banana. Disponível em: <http://www.unctad.info/en/Infocomm/A ACP-Products/COMMODITY-

PROFILE--- Banana/>. Acesso em Abr. de 2015. 